\title{
Shockwave-Based Automated Vehicle Longitudinal Control Algorithm for Nonrecurrent Congestion Mitigation
}

\author{
Liuhui Zhao, ${ }^{1}$ Joyoung Lee, ${ }^{2}$ Steven Chien, ${ }^{2,3}$ and Cheol $\mathrm{Oh}^{4}$ \\ ${ }^{1}$ Greenman-Pedersen, Inc., 21 W 38th St, New York, NY 10018, USA \\ ${ }^{2}$ John A. Reif, Jr. Department of Civil and Environmental Engineering, New Jersey Institute of Technology, \\ 17 Summit Street, Newark, NJ 07102, USA \\ ${ }^{3}$ School of Automobile, Chang'an University, Xian, Shaanxi, China \\ ${ }^{4}$ Department of Transportation and Logistics Engineering, Hanyang University at Ansan, 55 Hanyangdaehak-ro, \\ Sangnok-gu, Ansan-si, Gyeonggi-do 426-791, Republic of Korea \\ Correspondence should be addressed to Joyoung Lee; jo.y.lee@njit.edu
}

Received 12 December 2016; Revised 4 May 2017; Accepted 29 May 2017; Published 5 July 2017

Academic Editor: Richard S. Tay

Copyright (C) 2017 Liuhui Zhao et al. This is an open access article distributed under the Creative Commons Attribution License, which permits unrestricted use, distribution, and reproduction in any medium, provided the original work is properly cited.

\begin{abstract}
A shockwave-based speed harmonization algorithm for the longitudinal movement of automated vehicles is presented in this paper. In the advent of Connected/Automated Vehicle (C/AV) environment, the proposed algorithm can be applied to capture instantaneous shockwaves constructed from vehicular speed profiles shared by individual equipped vehicles. With a continuous wavelet transform (CWT) method, the algorithm detects abnormal speed drops in real-time and optimizes speed to prevent the shockwave propagating to the upstream traffic. A traffic simulation model is calibrated to evaluate the applicability and efficiency of the proposed algorithm. Based on $100 \% \mathrm{C} / \mathrm{AV}$ market penetration, the simulation results show that the CWT-based algorithm accurately detects abnormal speed drops. With the improved accuracy of abnormal speed drop detection, the simulation results also demonstrate that the congestion can be mitigated by reducing travel time and delay up to approximately $9 \%$ and $18 \%$, respectively. It is also found that the shockwave caused by nonrecurrent congestion is quickly dissipated even with low market penetration.
\end{abstract}

\section{Introduction}

Since the Intermodal Surface Transportation Efficiency Act (ISTEA) [1] launched in 1991, Intelligent Transportation System (ITS) has been deployed and expected to improve safety and mobility. However, the costs resulting from traffic congestion still increased by $50 \%$ over the previous decades and resulted in approximately 5.5 billion hours of extra delays and about 2.9 billion gallons of fuel wasted in the United States in 2011 [2]. Particularly, it is reported that total delays caused by nonrecurrent traffic congestions account for up to $70 \%[3]$.

Several years ago, an innovative concept in ITS was initiated so called Connected Vehicles (CV), formerly called Vehicle Infrastructure Integration (VII), in the United States. By enabling the communications for vehicle-to-vehicle (V2V) and vehicle-to-infrastructure (V2I), CV is expected to bring seamless benefits beyond the existing ITS applications. However, it is challenging to implement the applications due to lack of detail and accurate individual vehicular information. Under such a cooperative vehicle infrastructure environment, numerous cutting-edge applications focusing on improving safety and mobility have been proposed, and some of them have demonstrated promising performance.

With limited available resources for constructing greater capacity of road facilities and expanding the scale of road networks, ever-increasing traffic demand has made the transportation infrastructure systems more vulnerable to congestion caused by nonrecurrent events, such as traffic accidents. Unlike recurrent congestions, it is challenging to detect the time and location of a nonrecurrent event precisely and instantaneously with existing traffic sensing technology. Speed harmonization, achieved via variable speed limit control, could be applied to adjust the approaching vehicle speed 
based on the prevailing traffic conditions, which has proven itself an effective congestion mitigation solution approach. In the advent of the CV environment, the vehicles equipped with wireless communication devices are capable of capturing prevailing traffic conditions spatially and temporally. Therefore, it is not surprising to deploying them for accurately detecting nonrecurrent congestion in real-time. Meanwhile, with rapid advancement of real-time sensing and control technologies, automated vehicle (AV) has dragged great attention for its proven capability to dramatically improve roadway capacity. Coupled with CV technology, AV is expected to produce even greater benefits by rapidly harmonizing traffic flow to ease congestion.

This paper proposes a shockwave-based speed harmonization algorithm designed for manipulating longitudinal movements of $\mathrm{AVs}$ to suppress nonrecurrent congestion. Powered by the CV environment, individual vehicular speed and position information over space and time is captured in real-time. With the speed profile information, the proposed algorithm constructs shockwaves to detect nonrecurrent speed drops near the boundary of the congested area by employing continuous wavelet transform (CWT) method $[4,5]$. In turn, a speed control strategy is applied for the longitudinal maneuver of automated vehicles to eliminate the propagation of the shockwave. With a simulation test bed developed by using microscopic traffic simulation software VISSIM [6], we have conducted proof-of-concept (POC) tests to demonstrate the effectiveness of the proposed algorithm.

This paper is structured as follows. In the next section, the review of relevant literature and research efforts in the state-of-the-arts for real-time shockwave detection and speed harmonization are discussed. In the methodology section, an approach to handle shockwave detection is briefly discussed along with the CWT method and the speed control algorithm. Simulation-based evaluation studies are then presented in the section of proof-of-concept. Finally, the research findings and suggestions are concluded.

\section{Literature Review}

The majority of numerous research efforts on speed harmonization focused mainly on two objectives: improving roadway safety and enhancing traffic mobility. Concerning road safety, Lee et al. [7] investigated the impact of variable speed limit control on crash potential for the freeways, which indicated that speed control reduced crash potential by $5 \%-17 \%$ because speed limits were dynamically reduced subject to various levels of traffic conditions. A study conducted by Abdel-Aty et al. [8] aimed at improving freeway safety by setting a lower speed limit for upstream traffic and a higher speed limit for downstream traffic. A considerable reduction in crash potential under non-congested conditions was achieved; however no significant improvement are observed in case of heavily congested conditions.

In addition to the safety benefits which are commonly agreed by some of previous studies, others found that the mobility can be significantly improved with speed harmonization approaches. For instance, Carlson et al. [9] found that the total travel time can be reduced by $15.3 \%$ with optimal speed limit control. Hadiuzzaman and Qiu [10] proposed a cell transmission model based speed limit control on freeways, which yielded $10 \%-15 \%$ reduction in travel time and 5\%-7\% throughput increase under different control scenarios. While several factors (e.g., complexity of networks, level of compliance, and assessment methodology) may affect the contradictory findings in the literature, it was found that proactive models tend to yield greater benefits than reactive control algorithms. However, due to the requirements for further predictions and complexity to the traffic operators, proactive models have not been implemented in the field yet [11].

As mentioned in many simulation studies, speed harmonization could be effective in improving traffic mobility through resolving shockwaves. Hegyi et al. [12] demonstrated $21 \%$ reduction in total travel time through speed limit control aiming at eliminating shockwaves. Hadiuzzaman et al. [13] predicted the formation of a shockwave in the controlled area, which could activate an optimal coordinated speed harmonization system to reduce the impact and yield the minimum total travel time. With a distributed controller for each speed limit sign, Popov et al. [14] resolved shockwaves by optimizing controller parameters considering various levels of congestion.

In the advent of CV environment, Sun et al. [15] integrated the concept of cooperative vehicle infrastructure systems with the METANET model in which a predictive speed harmonization model was developed to suppress the propagation of shockwaves. Zhu and Ukkusuri [16] proposed a speed limit optimization model to estimate traffic state evolution and determine the optimal speed control for minimizing total travel time. Zheng et al. [5] also applied a CWT algorithm to analyze freeway traffic condition and identify bottlenecks based on the speed data collected from loop detectors. Talebpour et al. [4] embedded a CWT algorithm for detecting shockwaves and proposed a rule-based control algorithm based on traffic states (i.e., flow, density, and speed information). Both of the studies suggested that CWT is able to quickly capture abnormal speed changes and detect shockwaves.

Although many studies have focused on investigating speed harmonization for alleviating recurrent congestion, few have been conducted in a CV environment to ease nonrecurrent congestion. With individual vehicles' speed profile data collected from $\mathrm{C} / \mathrm{AVs}$, it is possible to dynamically detect congestion at any location, continuously manipulate longitudinal movements of AVs, and suppress shockwaves at early stages. Different from the previous studies, this paper investigates the influence of imperfect information on the effects of speed harmonization under different market penetration rates of equipped vehicles.

\section{Methodology}

3.1. Shockwave Detection. The CV environment enhances the existing technologies in collecting the speed profile of individual vehicles serving as an input for shockwave detection. In this study, the continuous wavelet transform (CWT) method is applied to identify the propagation of shockwaves based on vehicular speed profile [11]. 
The general formulation for CWT of a continuous signal $\chi(t)$ is represented as follows:

$$
\operatorname{CWT}(\alpha, \beta)=\omega(\alpha) \int_{-\infty}^{\infty} \chi(t) \psi\left(\frac{t-\beta}{\alpha}\right) d t
$$

where $\alpha$ is a scale parameter, $\beta$ is a shift parameter, and $\omega(\alpha)$ is the weighting function. The term $1 / \sqrt{\alpha}$ is commonly used to normalize energies produced at all scales. As discussed in Zheng and Washington [17], although different wavelets could be applied and provide similar results for various signals, the analysis results might be significantly influenced by the selected wavelet. To detect discontinuities and peaks in the traffic data, previous studies showed that Mexican Hat Wavelet is commonly used with better performance [11, 12 , 17]. Utilizing the research findings on the comparison of various wavelets, this paper treats the speed as the input signal to the wavelet transformation, and the Mexican Hat Wavelet is selected as the mother wavelet, represented as follows:

$$
\psi\left(\frac{t-\beta}{\alpha}\right)=\left[1-\left(\frac{t-\beta}{\alpha}\right)^{2}\right] e \frac{-((t-\beta) / \alpha)^{2}}{2} .
$$

Therefore, a wavelet transform of speed $v(t)$ is formulated by integrating (2) into (1),

$$
\begin{aligned}
& \operatorname{CWT}(\alpha, \beta) \\
& \quad=\frac{1}{\sqrt{\alpha}} \int_{-\infty}^{\infty} v(t)\left[1-\left(\frac{t-\beta}{\alpha}\right)^{2}\right] e \frac{-((t-\beta) / \alpha)^{2}}{2} d t
\end{aligned}
$$

Then the average wavelet-based energy at $\beta$ is calculated based on the CWT for different scales

$$
E(\beta)=\frac{1}{\max (\alpha)} \int_{0}^{\infty}|\operatorname{CWT}(\alpha, \beta)|^{2} d \alpha .
$$

It is worth noting that the vehicular speed profile data is aggregated for any freeway segment over time, so that the noise in the data could be reduced without loss of capturing sharp speed changes. The energy is then calculated and examined during the simulation for early shockwave detection. Figure 1 illustrates an example for the application of CWT to detect shockwaves under $100 \% \mathrm{C} / \mathrm{AV}$ market penetration with $200 \mathrm{ft}$ aggregated data at 10-second interval: (a) speed diagram over time of a segment; (b) CWT coefficient at $\alpha=$ 3; (c) contour of CWT coefficient with $\alpha$ varying from 1 to 6; (d) average energy across scales. Figure 1 shows that whenever significant change in the input speed occurs, it will be reflected in the coefficient, and these local minimum speeds will be represented in the energy as local maxima.

3.2. Speed Harmonization Strategy. At the end of each time step, the speed information is aggregated for each segment. If a drastic speed drop is detected or the speed is continuously decreasing for a predefined time period, a speed control strategy is triggered for the upstream segments. Then, the entire network will be checked and potential shockwaves as well as the locations of the bottlenecks will be identified. In turn, a speed control algorithm will be applied if necessary for speed harmonization and shockwave suppress. Otherwise, the control status will be switched off.

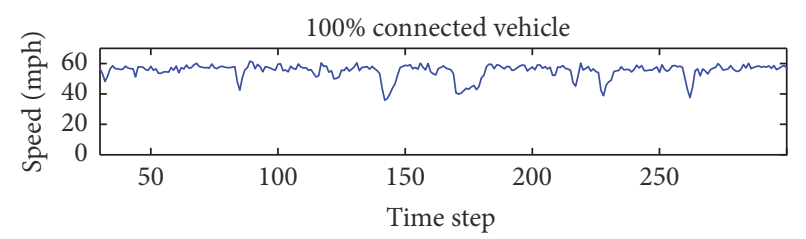

(a)

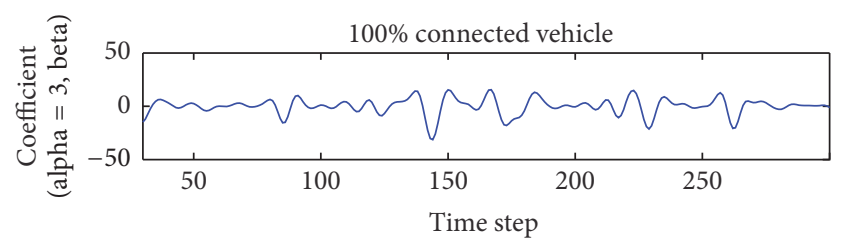

(b)

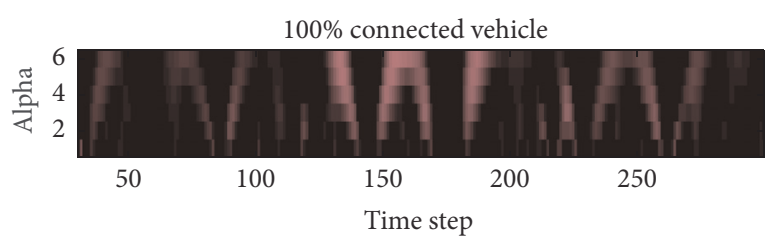

(c)

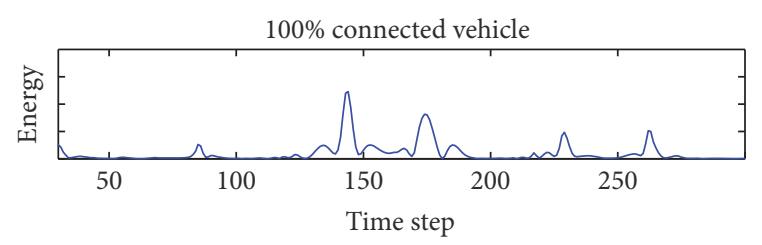

(d)

FIgURE 1: A sample of CWT algorithm.

Let $t$ be the time step, $s$ stands for a segment in the network, and $b$ stands for an index of bottlenecks. Assume that $v_{b}^{t}$ denotes the speed at bottleneck $b$ and $v_{s}^{t}$ denotes the average speed of segment $s$ at time $t . v_{\mathrm{sl}}$ represents the speed limit, and $v_{s}^{t+1}$ represents the desired speed for time step $t+1$. The control flow chart is illustrated in Figure 2. In POC study, speed data is collected every 10 seconds from equipped vehicles and aggregated by $200 \mathrm{ft}$ segment. After speed drop detection for each segment, bottlenecks are instantaneously determined where the speed is lower than those of its surrounding 20 segments (10 upstream, 10 downstream). Two speed control schemes including increasing and decreasing desired speed limit are applied to the upstream and downstream segments of a bottleneck, respectively. For the upstream segment, a maximum speed reduction of $8 \mathrm{mph}$ per time step is set to mitigate the speed change for safety concern and a minimum $5 \mathrm{mph}$ per time step is applied to effectively control the approaching speeds of upcoming vehicles.

\section{Proof-of-Concept Test}

A hypothetical microscopic traffic simulation network is created in VISSIM to test the proposed algorithm under different scenarios. The network consists of a 3-mile long 2lane basic freeway segment (i.e., without ramps) as shown in Figure 3. The design speed limit is $65 \mathrm{mph}$, and the average 


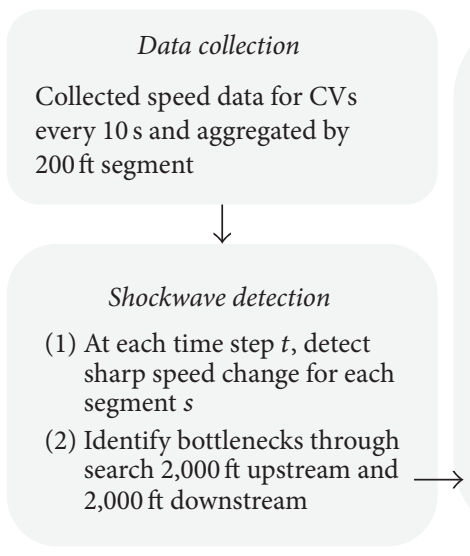

Speed harmonization

For each bottleneck $b$, a following control algorithm is applied in its upstream segments

(a) For upcoming vehicles within $2,000 \mathrm{ft}$,

FIGURE 2: Shockwave detection and speed harmonization flow chart.

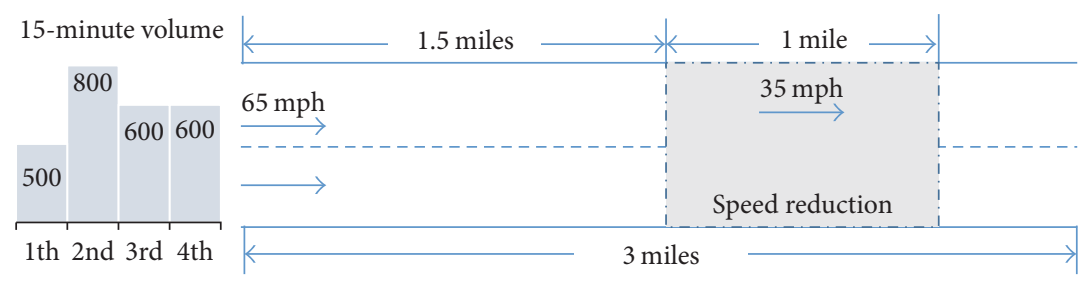

FIGURE 3: Proof-of-concept test network configuration.

hourly volume is $3000 \mathrm{vph}$. Hypothetical 15-minute fluctuated volumes are created as $2000 \mathrm{vph}, 4000 \mathrm{vph}, 3000 \mathrm{vph}$, and $3000 \mathrm{vph}$. A speed reduction zone is presented in the route at 1.5 mile with length of 1 mile, which provides a reduced speed limit at $35 \mathrm{mph}$. The total simulation time is 1 hour. The network is divided into $200 \mathrm{ft}$ segment, and the data collection interval is 10 seconds. In addition, the network is calibrated by adjusting parameters (i.e., standstill distance, minimum headway, and following variance) of Widemann-99 car following model to achieve the capacity of 2,200 veh/hour/lane (vphpl).

To capture vehicle information and investigate network performance, a set of varying $\mathrm{C} / \mathrm{AV}$ market penetration rates is applied in the test. Therefore, in the simulation model, two vehicle types are modeled, including C/AV and traditional vehicles. At each time interval, the individual vehicle information will be collected by $\mathrm{C} / \mathrm{AV}$, which will be aggregated based on predefined segments for traffic state estimation. If speed adjustments are necessary, C/AV will receive feedback and change their desired speeds accordingly during the following time step. While the information from traditional vehicles in the network cannot be collected, they are to be affected by surrounding C/AV and adjust their speeds following the traffic flow.

Three scenarios are created to test the proposed algorithm, including base case, speed drop case, and speed control case, which are described as follows. For each scenario, a total number of five simulation runs is conducted with different random seeds. The network performance in terms of average speed, travel time, and delay as well as average throughput is calculated for comparison. (i) Scenario 1 (Base Case). 3-mile freeway segment without speed reduction zone, an average of $3000 \mathrm{vph}$ is the input volume

(ii) Scenario 2 (Speed Drop Case). The same freeway segment and input volume as Scenario 1. 1-mile speed reduction zone is placed in the middle of the segment (starting at 1.5 mile). Within the speed reduction zone, the speed limit is dropped to $35 \mathrm{mph}$.

(iii) Scenario 3 (Speed Control Case). The same configuration as Scenario 2. However, in Scenario 3, realtime vehicular data is collected from C/AVs and the proposed speed harmonization algorithm is implemented in the network.

The overall network performance with and without speed reduction zone (i.e., Scenarios 1 and 2, resp.) is represented in Table 1 and speed diagram shown in Figure 4 for comparison. With given hourly volume setup under Scenario 1, the average speed of traveled vehicles is $53 \mathrm{mph}$, with an average delay of 14 seconds per vehicle. Because of increased volume from the second 15-minute interval, a minor speed drop can be observed. Thus, in the total 3-mile network, the total travel time is 161 hours with 2,737 vehicles arrived per hour. In comparison with Scenario 1, the average speed dropped by $28 \%$ and the number of arrived vehicles reduced by $2 \%$ when a speed reduction zone is presented.

4.1. Shockwave Detection Results. An example of speed and corresponding energy for three consecutive links is shown in Figure 5, with 10-second data collection interval. Because of the presence of speed reduction zone and increasing volume, significant speed drops occur between time steps 100 and 200. 
TABLE 1: Network performance with and without a speed reduction zone.

\begin{tabular}{lcc}
\hline Performance measure & $\begin{array}{c}\text { Scenario 1 } \\
\text { (base case) }\end{array}$ & $\begin{array}{c}\text { Scenario 2 } \\
\text { (speed drop case) }\end{array}$ \\
\hline Average speed (mph) & 53 & 38 \\
Average delay per vehicle (seconds) & 14 & 37 \\
Total travel time (hours) & 161 & 222 \\
Throughput (vph) & 2,737 & 2,676 \\
\hline
\end{tabular}
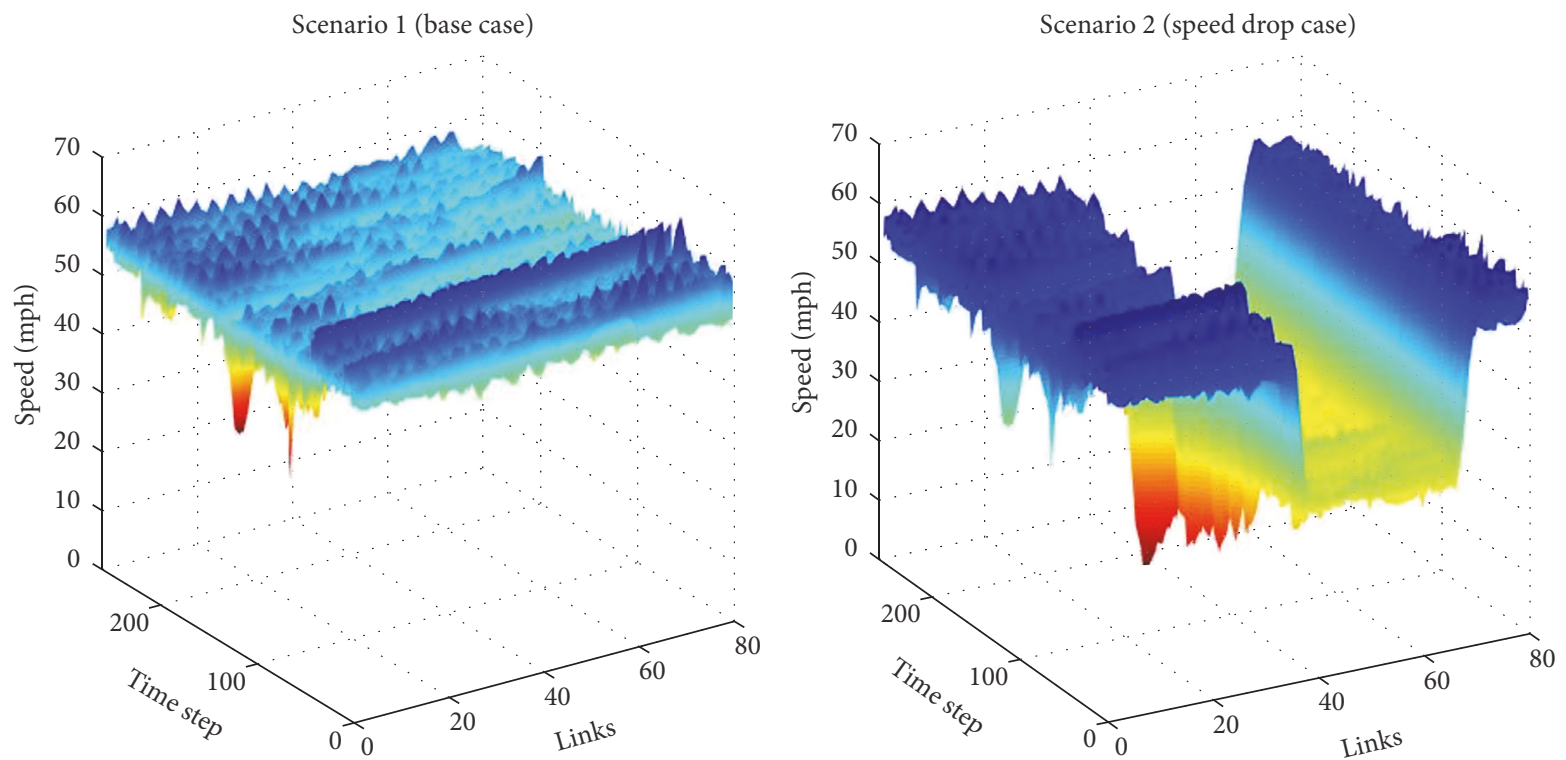

FIGURE 4: Speed comparison under normal condition and with a speed reduction zone.

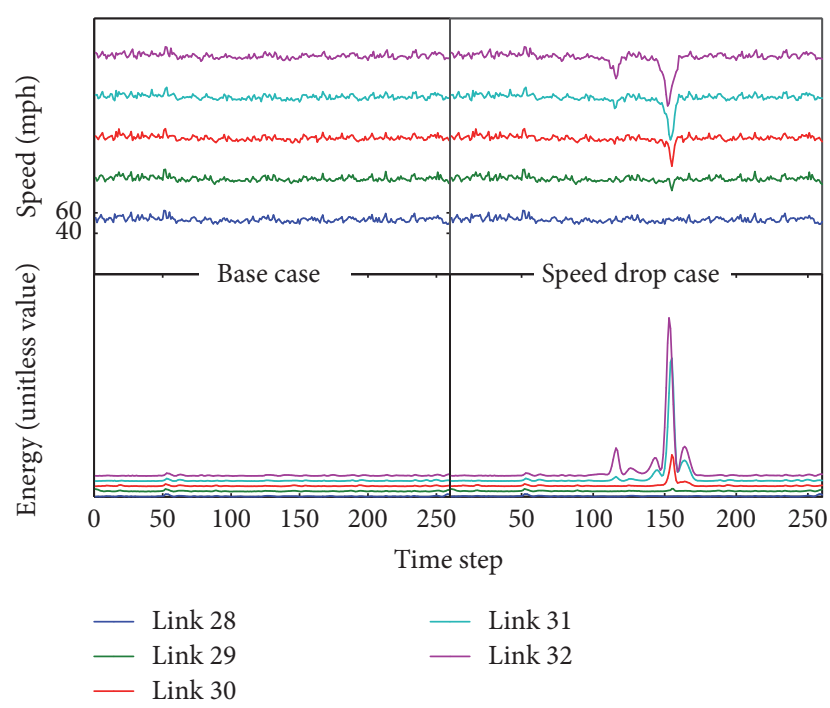

FIGURE 5: A comparison of speed and corresponding energy under Scenarios 1 and 2 .

While link 28 locates upstream and link 32 downstream of the route, shockwave propagation can be observed in each of the speed and energy diagrams.

In order to test the applicability of the CWT algorithm under low market penetration rate, a varying $\mathrm{C} / \mathrm{AV}$ rate from $10 \%$ to $100 \%$ is applied in the test network. Due to missing

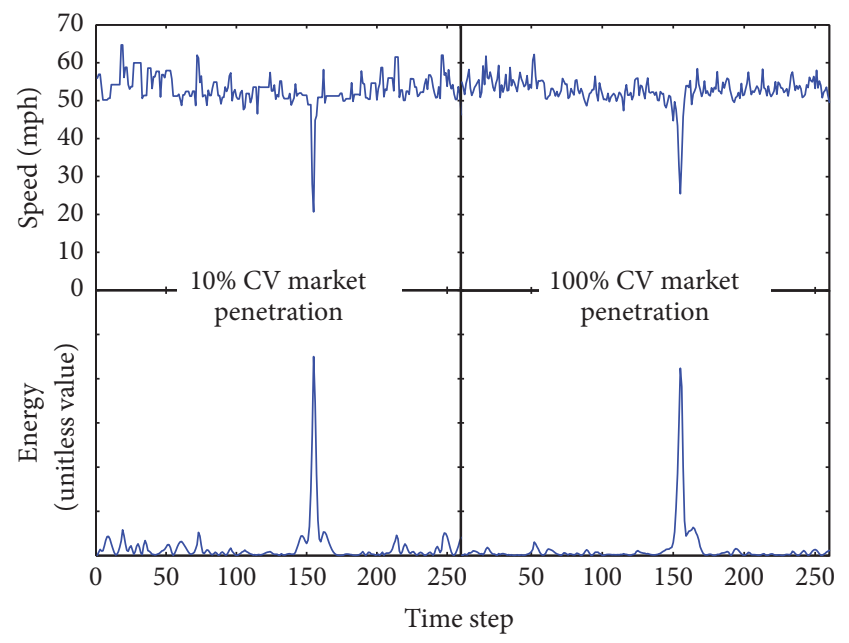

FIGURE 6: Speed and energy comparison for a single link.

data under low market penetration, speed adjustment was conducted so that speed from previous time step reserves at the no-data time step. Under low market penetration, imperfect information to detect speed drop is preserved in the high speed zone but more noises can be observed in the low speed zone, compared to $100 \%$ market penetration (e.g., Link 30 in Figure 6). However, looking into the energy diagram for both low (i.e., 10\%) and high (i.e., 100\%) market penetration 

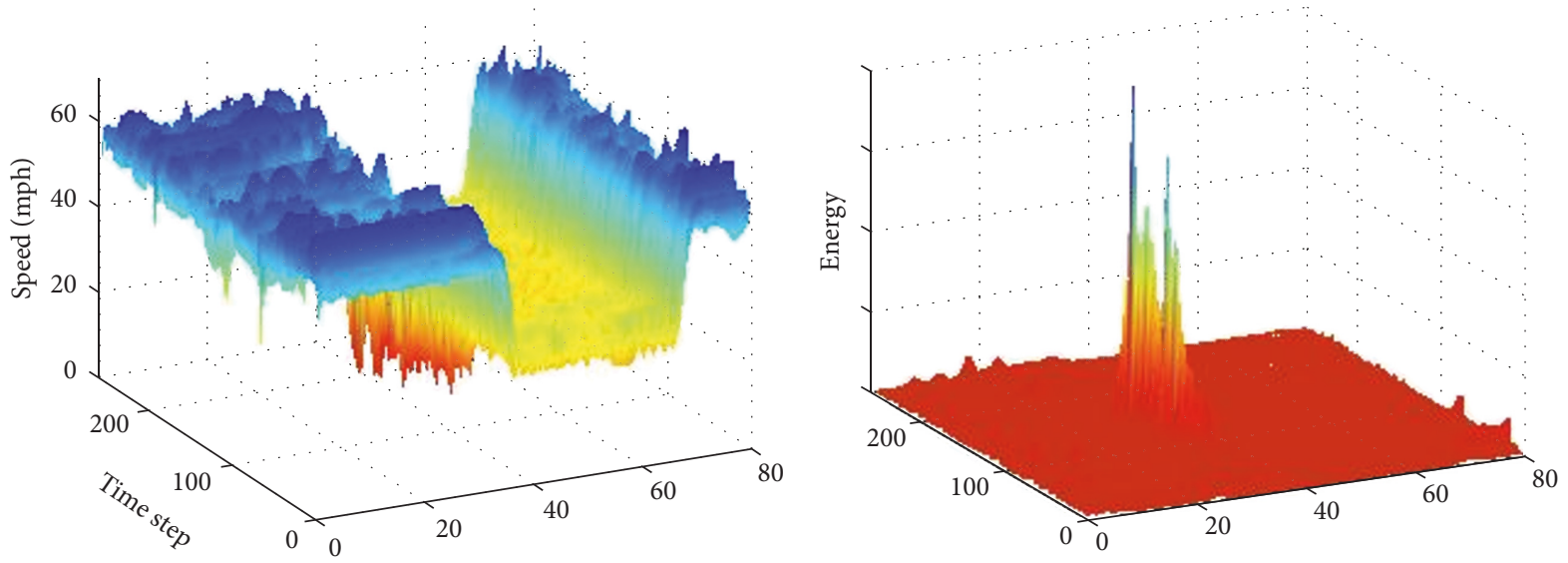

$10 \%$ CV market penetration
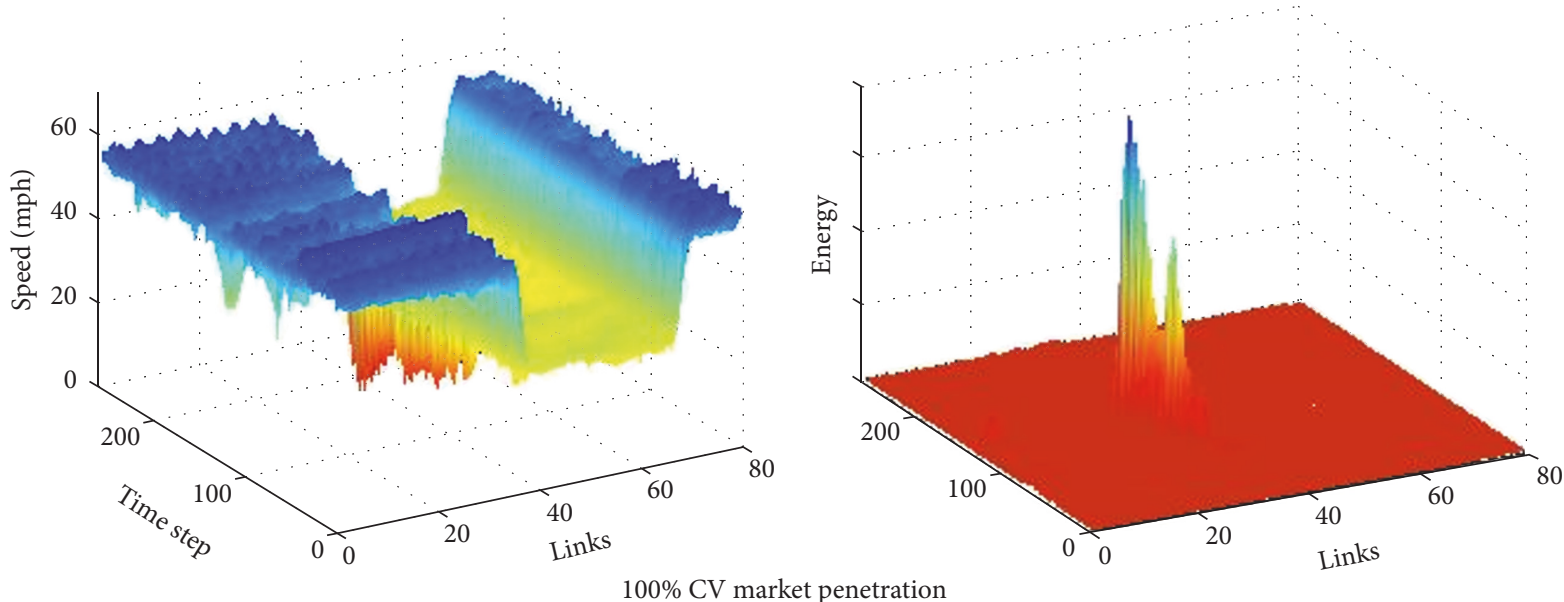

FIGURE 7: Speed and energy comparison under different market penetration rates.

rates, the speed drops as well as shockwaves over segments are well captured by energy pulses as shown in Figure 7. This finding clearly indicates that the proposed CWT algorithm is able to maintain the robustness for congestion detection regardless of market penetration rates of C/AV.

4.2. Speed Harmonization Results. With $100 \% \mathrm{C} / \mathrm{AV}$ market penetration rate, the effect of speed harmonization is summarized in Table 2, where Scenario 2 represents uncontrolled network at presence of speed reduction zone, and Scenario 3 represents controlled network. After speed control, the average speed is improved by $4 \mathrm{mph}$. Paired test shows that, at 95\% confidence level, the mean speed in controlled network is statistically greater than that in uncontrolled network. Although the average delay per vehicle is increased by 8 seconds, the overall travel time is significantly reduced (by 9\%) with more vehicles arrived at the destination (i.e., 17 additional vehicles per hour).

As shown in Figure 8(a), the network could be separated into three zones for further analysis: the upstream of the speed reduction zone (Zone 1), the speed reduction zone (Zone 2), and the downstream of the reduction zone (Zone 3 ). It is clear that the control strategy not only increases the speeds of Zones 1 and 3 but also mitigates shockwaves on the boundaries between Zones 1 and 2. Within Zone 2, the speed over space and time is significantly smoothed. To get a closer observation of the shockwave due to speed reduction zone, Figure 8(b) enlarged the critical area in the time-space diagram in Figure 8(a). The $x$-axis and $y$-axis are labeled by time (minutes) and distance (miles), respectively. Observed from Figure 8(b), under Scenario 2 without controlling, the maximum length of shockwave is about 0.4 miles, around the 25th minute, whereas under Scenario 3 with controlling, not only is a large portion of shockwaves eliminated, but also the maximum length of shockwave is also reduced to a quarter mile.

With the vehicular data collected every 10 seconds for each segment in the simulation model, the boxplots in Figure 9 show the speed results of the three zones for each scenario: base case, uncontrolled speed case under speed drop, and controlled speed case under speed drop. It is discovered that, for Zone 1, the speed harmonization algorithm increased the average speed and also curbed the influence of speed reduction with less low speed cases. Within Zone 2, more vehicles were able to travel at a higher speed compared to uncontrolled network, through simultaneously 
TABLE 2: Network performance for Scenarios 2 and 3 under 100\% C/AV market penetration.

\begin{tabular}{lccc}
\hline Performance measure & $\begin{array}{c}\text { Scenario 2 (speed drop case W/O } \\
\text { harmonization) }\end{array}$ & $\begin{array}{c}\text { Scenario 3 (speed control case } \\
\text { W/harmonization) }\end{array}$ & Improvement (\%) \\
\hline Average speed (mph) & 38 & 42 & 10.5 \\
Average delay per vehicle (seconds) & 45 & 37 & 17.8 \\
Total travel time (hours) & 222 & 203 & 8.6 \\
Throughput (vph) & 2,676 & 2,693 & 0.6 \\
\hline
\end{tabular}

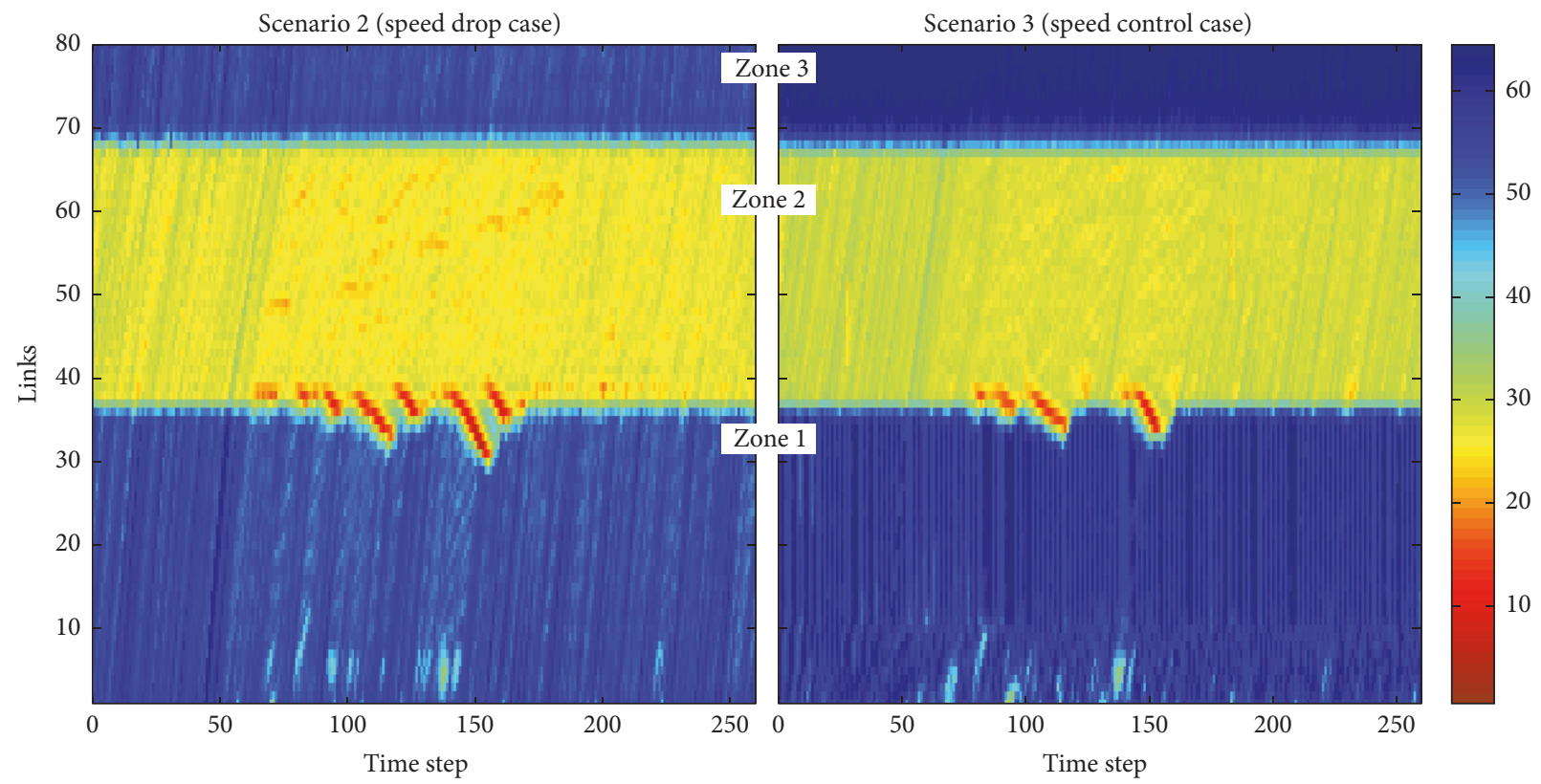

(a)

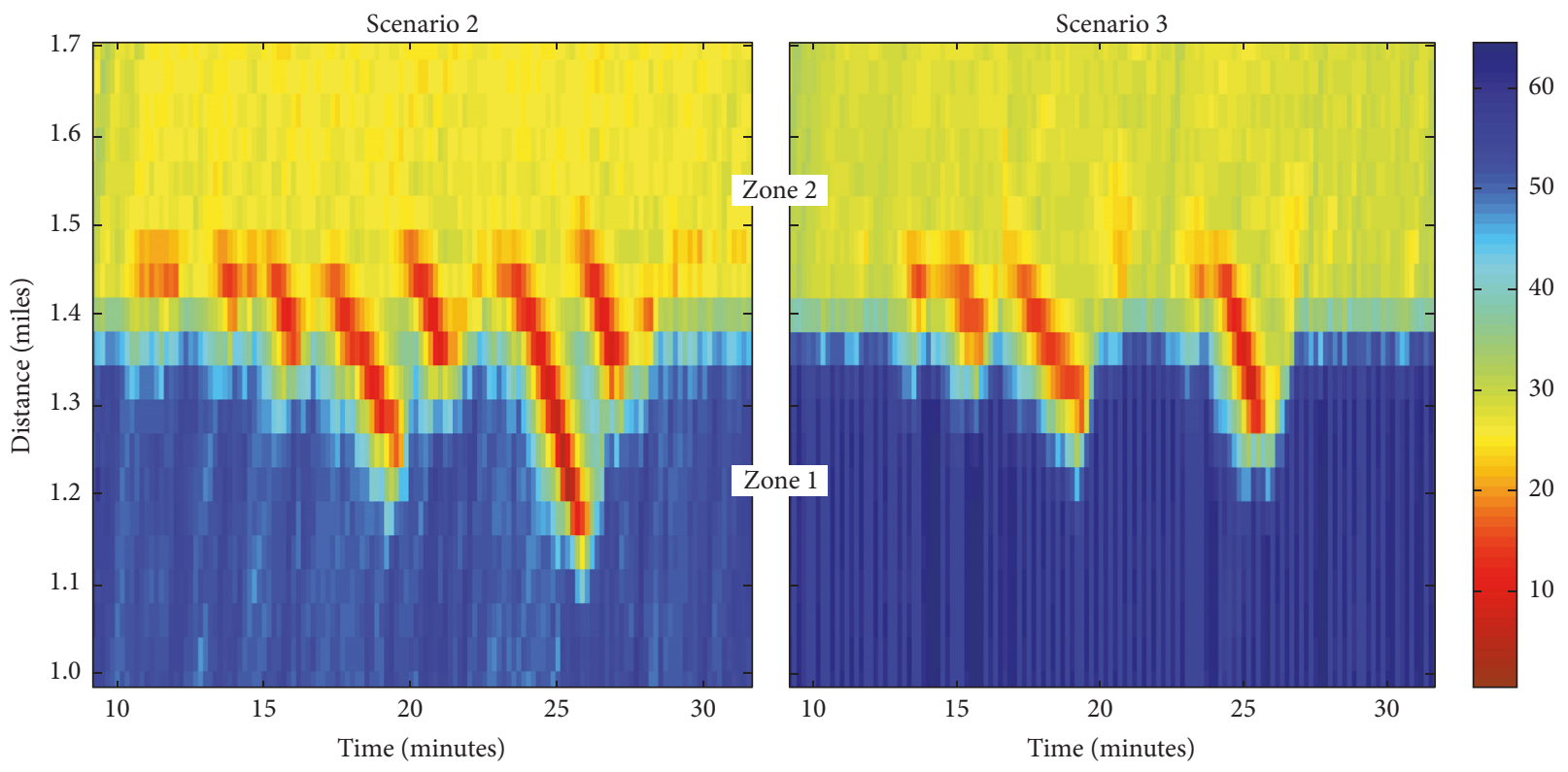

(b)

FigURE 8: Speed profiles under 100\% market penetration (Scenario 2 versus Scenario 3). 
TABLE 3: Mean speeds under different scenarios.

\begin{tabular}{|c|c|c|c|c|}
\hline & \multirow{2}{*}{ Scenario } & \multicolumn{3}{|c|}{ Zone } \\
\hline & & 1 (1.4 miles) & 2 (1.1 miles) & 3 (0.5 miles) \\
\hline \multirow{3}{*}{ Mean speed (stdev.) } & 1 (base case) & $54(2.6)$ & $53(2.3)$ & $55(2.4)$ \\
\hline & 2 (speed drop case) & $52(5.9)$ & $26(1.7)$ & $53(5.0)$ \\
\hline & 3 (speed control case) & $55(6.3)$ & $30(1.9)$ & $56(3.8)$ \\
\hline
\end{tabular}

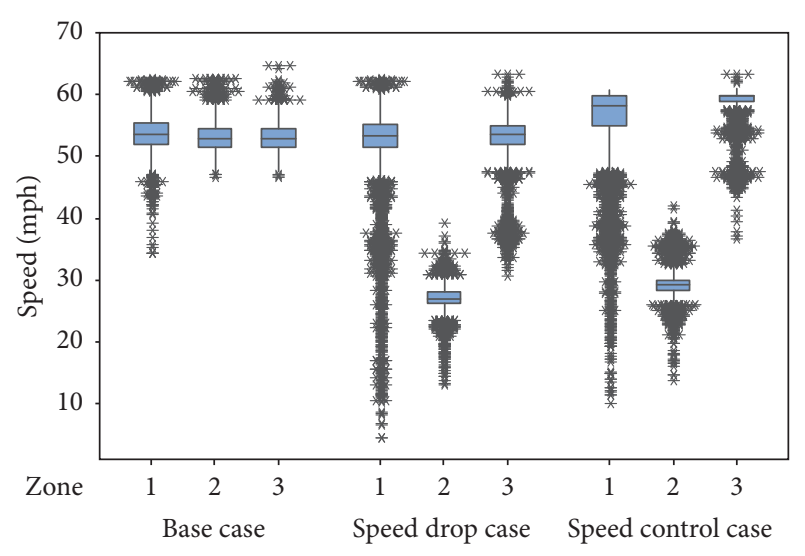

FIGURE 9: Speed distributions under different scenarios (100\% market penetration).

reducing upstream speed and increasing downstream speed. Therefore, in Zone 3 (downstream), a higher average speed can be achieved. The mean speed for each zone under three scenarios is summarized in Table 3.

The effects of speed harmonization under different C/AV market penetration rates and the associated network performance measures are shown in Figures 10 and 11, respectively. As the market penetration rate increases, the control algorithm can better suppress the shockwaves induced by speed reduction zone. Illustrated by speed distribution over three zones, under low market penetration (10\% 40\%), increasing the percentage of $\mathrm{C} / \mathrm{AV}$ s leads to better controlled backwards and forwards shockwaves (i.e., lower speed cases in both Zone 1 and Zone 3). Keeping increasing the rate of C/AVs, low speed cases are increased in the upstream zone, while reducing in Zone 3.

Looking into the network performance with speed control under different market penetration rates in Figure 11(a), the congestion impact seems to be eased, indicated by increased average speed and lowered travel time with increased C/AVs in the network. Compared to Scenario 2 (i.e., uncontrolled speed under speed drop), the throughput is heightened under all market penetration rates except for the $10 \%$ market penetration case. The average speed appears improved by $3 \mathrm{mph}$ with over $90 \% \mathrm{C} / \mathrm{AV}$ market penetration rates, $2 \mathrm{mph}$ with over $70 \%$, and $1 \mathrm{mph}$ with over $40 \%$. As a result, if the C/AVs take over $40 \%$ of all the vehicles, a reduction of total travel time by $2 \%$ can be yielded through the speed control. If $90 \%$ of the vehicles are C/AVs, a $5 \%$ reduction in the travel time can be expected.

A comparison has been conducted for the network performance under different speed control conditions. While the

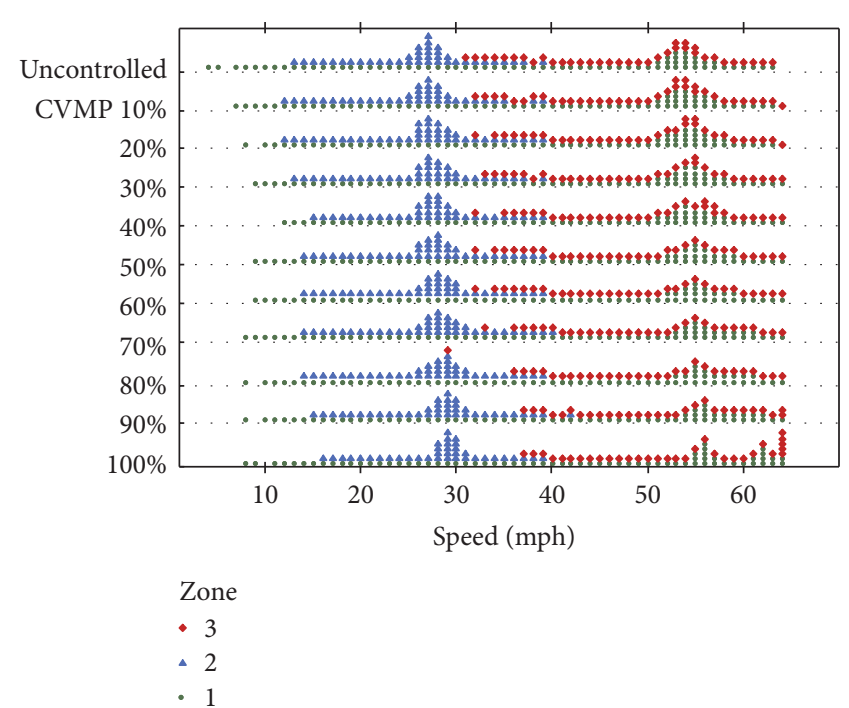

Figure 10: Comparison of speed distributions under different $\mathrm{C} / \mathrm{AV}$ market penetration rates. Each symbol represents up to 582 observations.

base case scenario represents speed data collection only from $\mathrm{C} / \mathrm{AV}$ s and speed control only on C/AVs (as discussed above), the compared strategy collects speed only from C/AVs but controls speed for all vehicles in the network based on the same shockwave detection and speed control algorithm. The network performance with such strategy is illustrated in Figure 11(b). As it shows, even with limited speed information due to low $\mathrm{C} / \mathrm{AV}$ market penetration, the proposed algorithm could still be effective in speed harmonization in comparison with $100 \%$ market penetration. The average speed is significantly improved with reduced total travel time even under $10 \% \mathrm{C} / \mathrm{AV}$ market penetration rate.

\section{Concluding Remarks}

This paper presented a proof-of-concept study of an innovative shockwave-based speed harmonization algorithm for the longitudinal control of automated vehicles under the $\mathrm{C} / \mathrm{AV}$ environment. The shockwave detection method based on continuous wavelet transform was proposed and evaluated along with a control algorithm for speed harmonization. Simulation-based evaluations have been conducted with a hypothetical VISSIM network handling a freeway segment with speed reduction zone to examine the applicability of the proposed algorithm.

The results showed that the applied algorithm detects sharp speed drop over time and shockwaves over space 


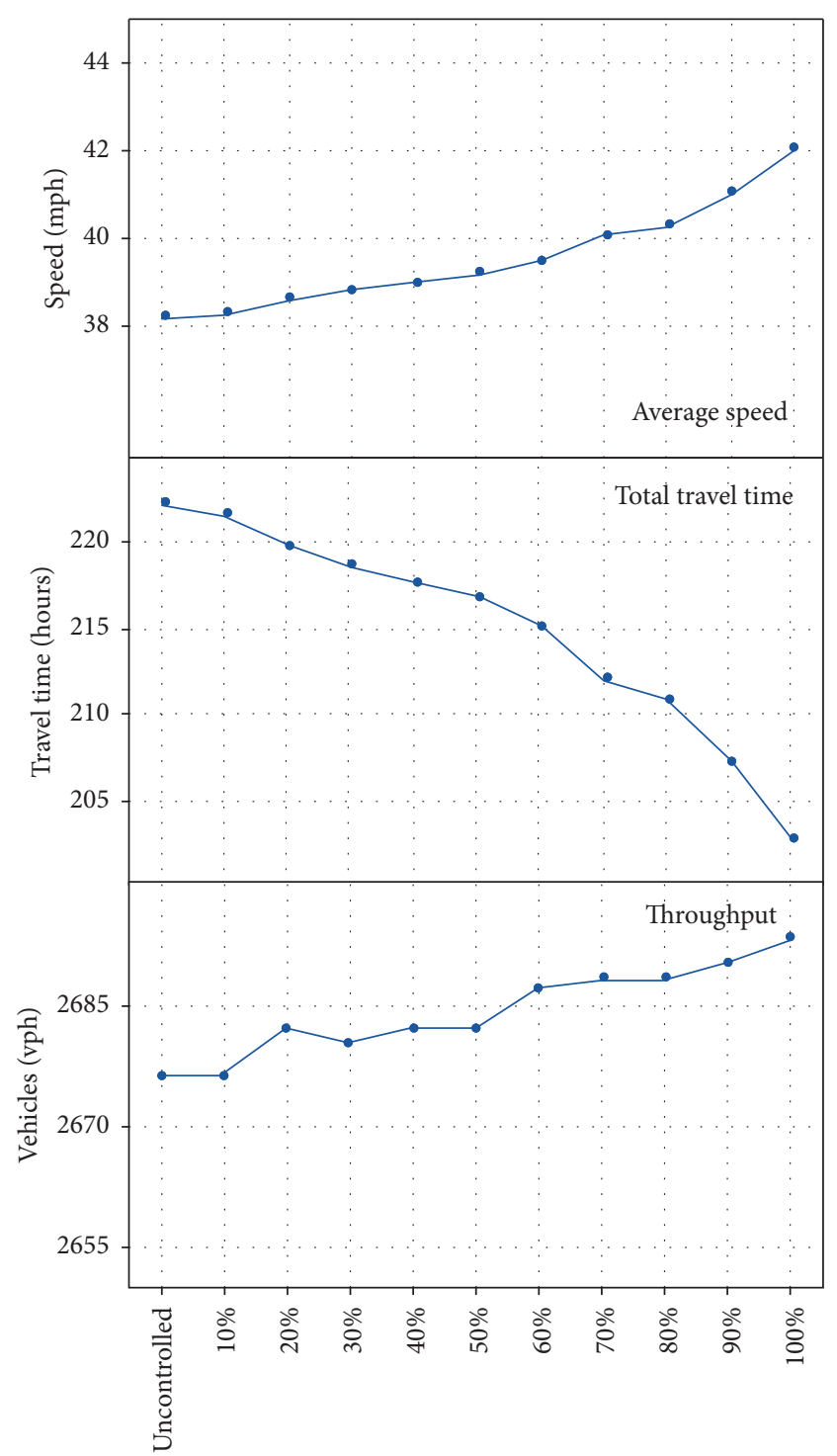

(a)

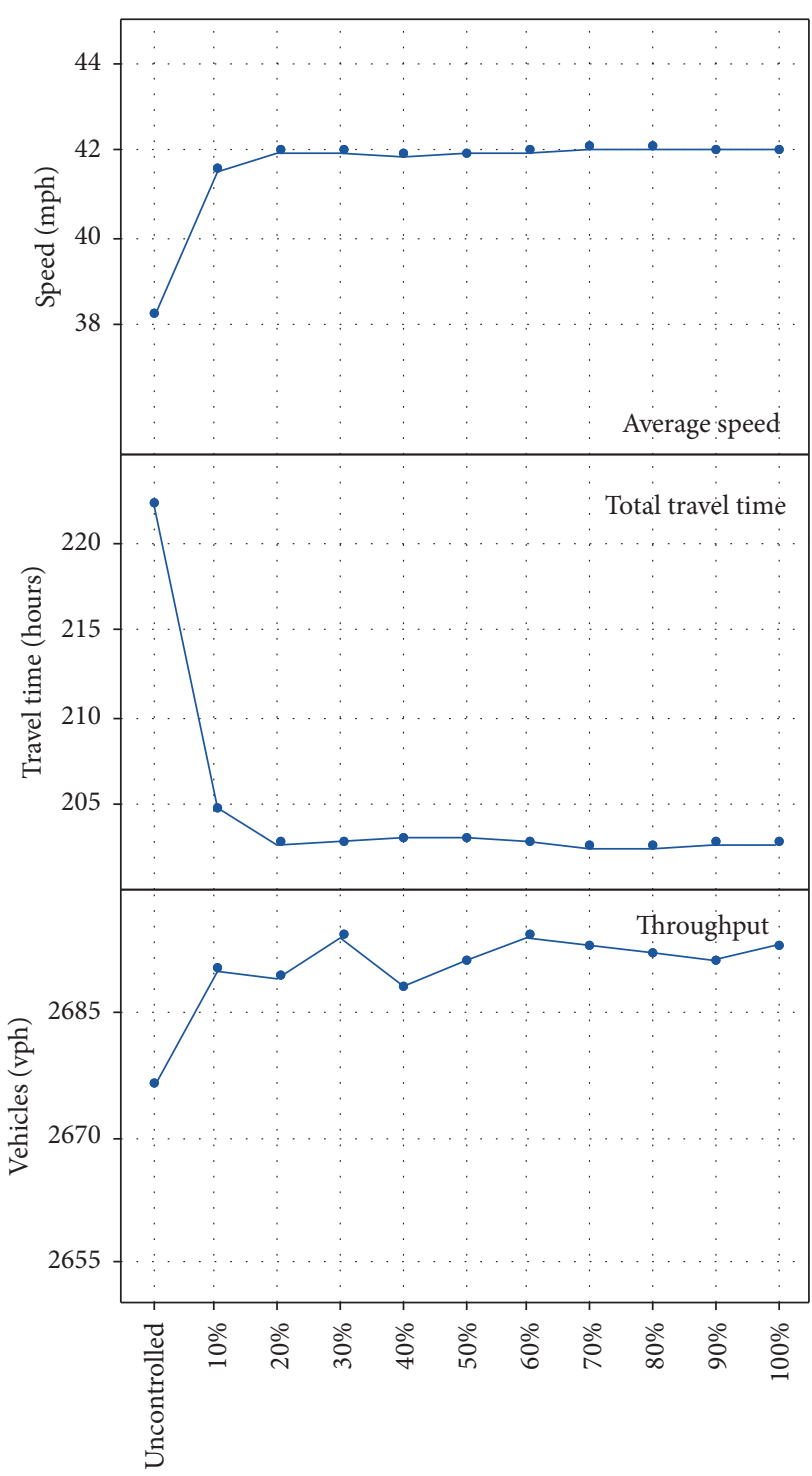

(b)

FIGURE 11: Network performance versus C/AV market penetration with different speed control strategies.

in real-time. Under $100 \% \mathrm{C} / \mathrm{AV}$ market penetration, it was discovered that the proposed speed control algorithm significantly improves the average speed compared to the uncontrolled speed case with the presence of a speed reduction zone. The network throughput also appeared increasing while reducing total travel time. By varying the rate of $\mathrm{C} / \mathrm{AV}$ market penetration from $10 \%$ to $100 \%$, the influence on speed harmonization was also investigated. The comparison of network performance and speed distribution revealed that the propagation of shockwaves to the upstream zone is effectively suppressed even under lower market penetration rates while the improvements of travel speed and total travel time appear marginal.

In this proof-of-concept test, the freeway in the simulation model is arbitrarily divided into multiple segments for traffic measure aggregation and shockwave detection, which can be easily relaxed with the communications of C/AV in the network. The immediate extension of this research would investigate the efficiency of detecting dynamic shockwave in real-time. Other extensions will focus on (1) developing a proactive speed harmonization algorithm to improve control effectiveness with low percentage of $\mathrm{C} / \mathrm{AV}$ and (2) investigating the applications of $\mathrm{C} / \mathrm{AV}$ on speed harmonization and its comparison with traditional vehicles. The proposed algorithm performance discussed in this study will be evaluated based on a larger scale simulation network under various rates of $\mathrm{C} / \mathrm{AV}$ market penetration.

\section{Conflicts of Interest}

The authors declare that they have no conflicts of interest. 


\section{Acknowledgments}

This work was in part supported by the National Research Foundation of Korea (NRF) grant funded by the Ministry of Science, ICT and Future Planning (MSIP) of Korea (NRF2010-0028693).

\section{References}

[1] "Intermodal Surface Transportation Efficiency Act (ISTEA)," http://ntl.bts.gov/DOCS/istea.html.

[2] D. Schrank, B. Eisele, and T. Lomax, "TTI's 2012 Urban Mobility Report," Texas Transportation Institute (TTI), College Station, TX, 2009.

[3] J. Short, "Measuring Recurring and Non-Recurring Congestion," in Proceedings of the NATMEC Conference, 2012, http:// onlinepubs.trb.org/onlinepubs/conferences/2012/NATMEC/ Short.pdf.

[4] A. Talebpour, H. Mahmassani, and S. Hamdar, "Speed harmonization," Transportation Research Record, no. 2391, pp. 69-79, 2013.

[5] Z. Zheng, S. Ahn, D. Chen, and J. Laval, "Applications of wavelet transform for analysis of freeway traffic: Bottlenecks, transient traffic, and traffic oscillations," Transportation Research Part B: Methodological, vol. 45, no. 2, pp. 372-384, 2011.

[6] VISSIM Version 5.40 User Manual PTV Planning Transport, Verkehr AG, Karlsruhe, Germany, 2011.

[7] C. Lee, B. Hellinga, and F. Saccomanno, "Evaluation of variable speed limits to improve traffic safety," Transportation Research Part C: Emerging Technologies, vol. 14, no. 3, pp. 213-228, 2006.

[8] M. Abdel-Aty, J. Dilmore, and A. Dhindsa, "Evaluation of variable speed limits for real-time freeway safety improvement," Accident Analysis and Prevention, vol. 38, no. 2, pp. 335-345, 2006.

[9] R. C. Carlson, I. Papamichail, and M. Papageorgiou, "Local feedback-based mainstream traffic flow control on motorways using variable speed limits," IEEE Transactions on Intelligent Transportation Systems, vol. 12, no. 4, pp. 1261-1276, 2011.

[10] M. Hadiuzzaman and T. Z. Qiu, "Cell transmission model based variable speed limit control for freeways," Canadian Journal of Civil Engineering, vol. 40, no. 1, pp. 46-56, 2013.

[11] D. Li, P. Ranjitkar, and A. Ceder, "A logic tree based algorithm for variable speed limit controllers to manage recurrently congested bottlenecks," in Proceedings of the Transportation Research Board 93rd Annual Meeting.

[12] A. Hegyi, B. de Schutter, and J. Hellendoorn, "Optimal coordination of variable speed limits to suppress shock waves," IEEE Transactions on Intelligent Transportation Systems, vol. 6, no. 1, pp. 102-112, 2005.

[13] M. Hadiuzzaman, J. Fang, Y. Luo, and T. Z. Qiu, "Evaluating performance of a proactive optimal variable speed limit control using different objective functions," Procedia-Social and Behavioral Sciences, vol. 96, pp. 2895-2906, 2013.

[14] A. Popov, A. Hegyi, R. Babuška, and H. Werner, "Distributed controller design approach to dynamic speed limit control against shockwaves on freeways," Transportation Research Record, no. 2086, pp. 93-99, 2008.

[15] R. Sun, J. Hu, X. Xie, and Z. Zhang, "Variable speed limit design to relieve traffic congestion based on cooperative vehicle infrastructure system," Procedia-Social and Behavioral Sciences, vol. 138, pp. 427-438, 2014.
[16] F. Zhu and S. V. Ukkusuri, "Accounting for dynamic speed limit control in a stochastic traffic environment: a reinforcement learning approach," Transportation Research Part C: Emerging Technologies, vol. 41, pp. 30-47, 2014.

[17] Z. Zheng and S. Washington, "On selecting an optimal wavelet for detecting singularities in traffic and vehicular data," Transportation Research Part C: Emerging Technologies, vol. 25, pp. 18-33, 2012. 


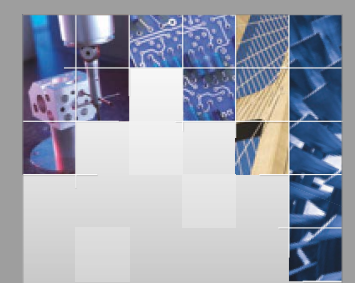

\section{Enfincering}
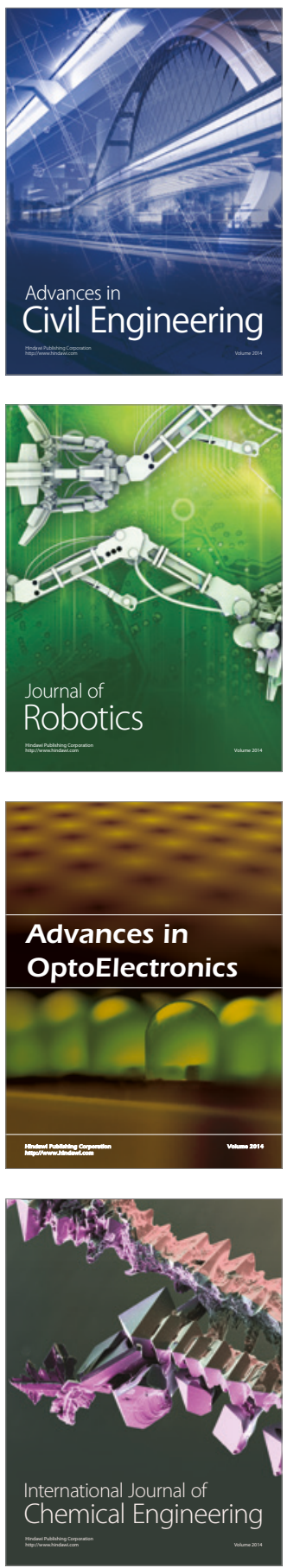

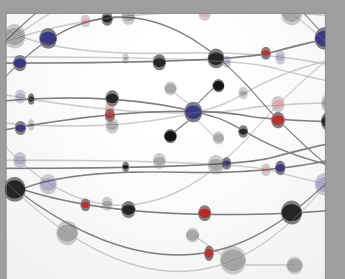

The Scientific World Journal

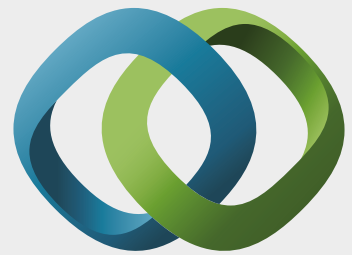

\section{Hindawi}

Submit your manuscripts at

https://www.hindawi.com
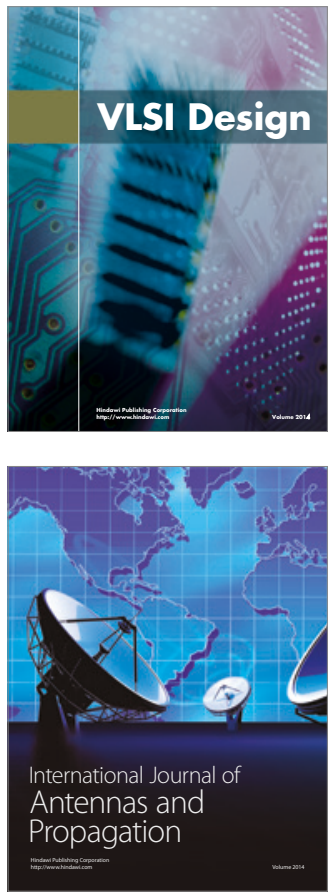

\section{Rotating}

Machinery
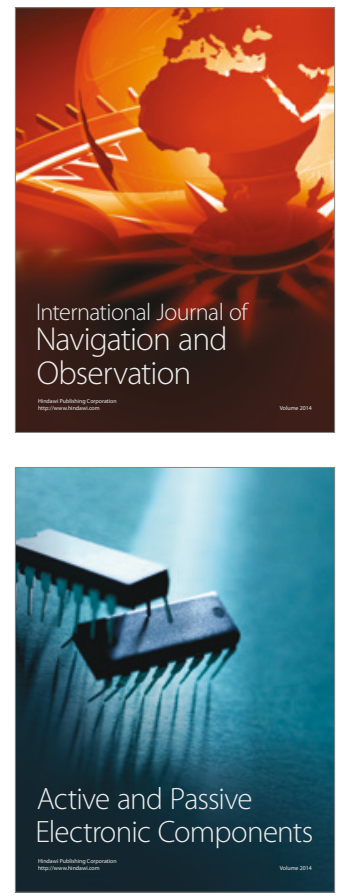
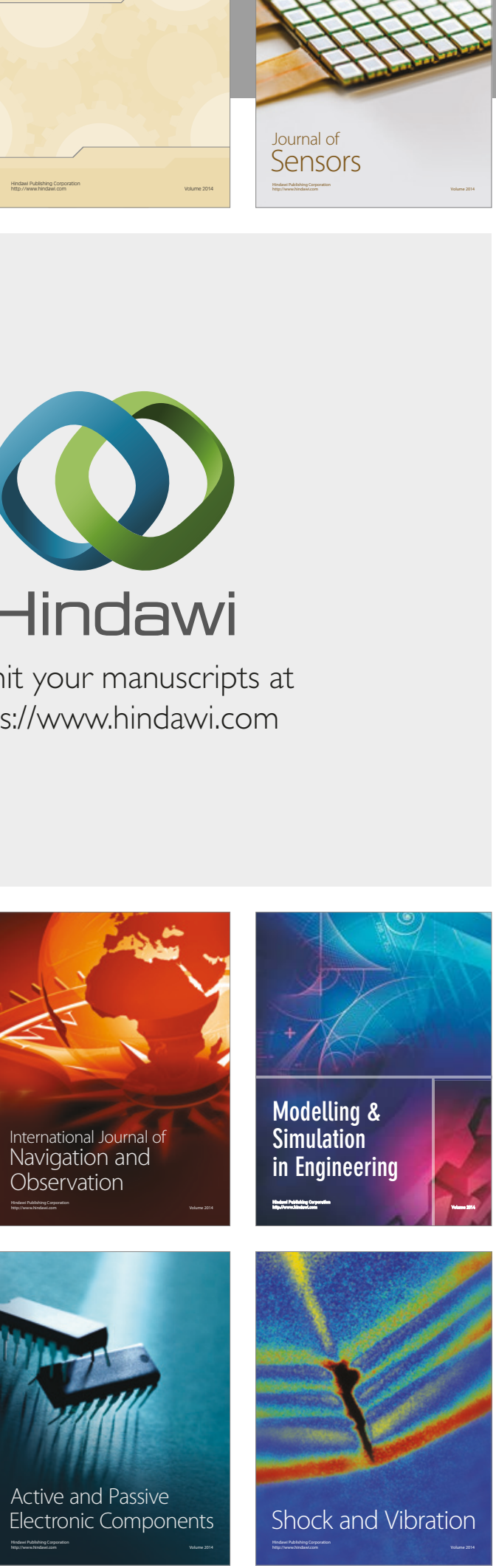
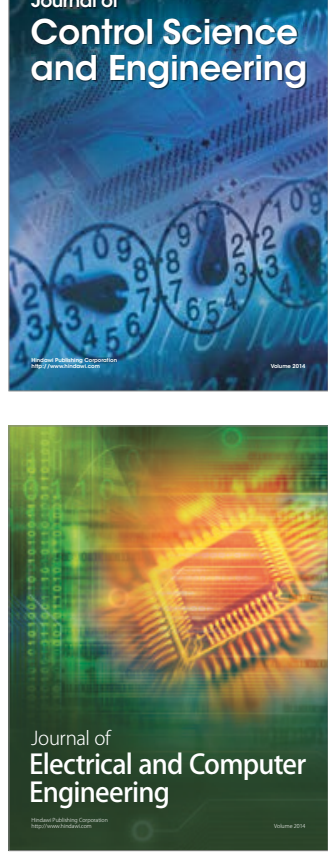

Distributed

Journal of

Control Science

and Engineering
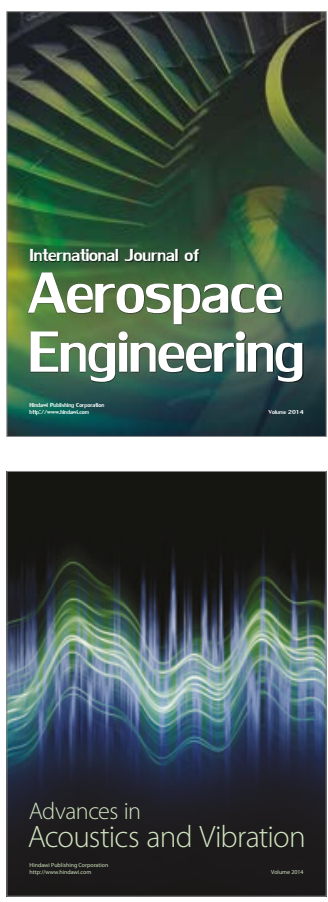

Sensor Networks 